\title{
SiPhotonics/GaAs 28-GHz Transceiver With Reflective EAM for Laser-Less mmWave-Over-Fiber
}

\author{
Laurens Bogaert ${ }^{\circledR}$, Joris Van Kerrebrouck ${ }^{\circledR}$, Haolin Li ${ }^{\circledR}$, Igor Lima de Paula ${ }^{\circledR}$, Kasper Van Gasse ${ }^{\circledR}$, \\ Chia-Yi Wu ${ }^{\circledR}$, Peter Ossieur, Sam Lemey ${ }^{\circledR}$, Hendrik Rogier ${ }^{\circledR}$, Piet Demeester ${ }^{\circledR}$, Gunther Roelkens ${ }^{\circledR}$, \\ Johan Bauwelinck ${ }^{(0)}$, and Guy Torfs ${ }^{(1)}$
}

(Post-Deadline Paper)

\begin{abstract}
Exploring mmWave frequencies and adopting smallcell architectures are two key enablers for increased wireless data rates. To make these evolutions economically viable, centralized architectures based on radio-over-fiber (RoF) are devised. To reduce the complexity of the cellular network even further, RF-over-Fiber transmission schemes are adopted in combination with reflective uplink operation. This paper relies on a very low complexity narrowband GaAs electronics / Si photonics transceiver for scalable RFoF architectures with which we demonstrate a fiber-wireless link capable of transmitting over $7 \mathrm{~Gb} / \mathrm{s}$ in down- and uplink for a $2 \mathrm{~km}$ fiber and $5 \mathrm{~m}$ wireless link in the $28 \mathrm{GHz}$ band. Furthermore, it is shown that Rayleigh degradation caused by reflective uplink operation can be avoided by using a coherent detection scheme.
\end{abstract}

Index Terms-Millimeter-wave, narrowband, optoelectronic, radio-over-fiber, silicon photonics.

\section{INTRODUCTION}

$\mathbf{S}$ IGNIFICANT changes in the underlying mobile network are required to keep up with the ever increasing demand for high speed wireless data [1]. There are a multitude of solutions envisioned to boost the data capacity of the wireless infrastructure [2]. A first key enabler is the migration to mmWave frequencies, in particular to the licensed $28 \mathrm{GHz}$ band. This increases the available contiguous bandwidth and benefits from a significantly

Laurens Bogaert, Kasper Van Gasse, and Gunther Roelkens are with the Department of Information Technology (INTEC), Photonics Research Group, Ghent University-imec, Ghent 9052, Belgium (e-mail: laurens.bogaert@ugent.be; kasper.vangasse@ugent.be; gunther.roelkens@ ugent.be).

Joris Van Kerrebrouck, Haolin Li, Igor Lima de Paula, Chia-Yi Wu, Peter Ossieur, Sam Lemey, Hendrik Rogier, Piet Demeester, Johan Bauwelinck, and Guy Torfs are with the Department of Information Technology (INTEC), IDLab, Ghent University-imec, Ghent 9052, Belgium (e-mail: joris.vankerrebrouck@ugent.be; haolin.li@ugent.be; igor.limadepaula@ugent.be; chiayi.wu@ugent.be; peter.ossieur@ugent.be; sam.lemey@ugent.be; hendrik.rogier@ugent.be; piet.demeester@ugent.be; johan.bauwelinck@ugent.be; guy.torfs@ugent.be).

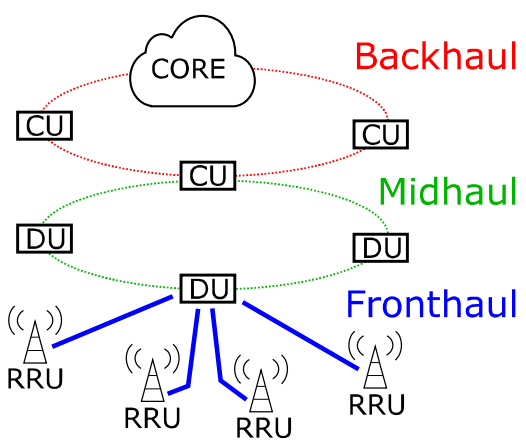

Fig. 1. Next-Generation Radio Access Network (NG-RAN) - CU: Central Unit; DU: Distributed Unit; RRU: Remote Radio Unit - This paper focuses on the fronthaul.

less congested spectrum than the nowadays often employed sub-6 GHz band. A second major change is the densification of the network. Such a small-cell approach boosts the overall data capacity of the network but suffers from scalability issues. Therefore, a centralized approach is key in future small-cell configurations to ensure, on the one hand, that the high-complexity functionalities, such as generation and processing of the RF signal, are performed at a central location. The remote radio units (RRUs) should, on the other hand, be mass-deployable and, therefore, be as cost-effective as possible. Next-generation radio access networks (NG-RAN) [3], [4] consist of central units (CUs) which are connected to the core network via the backhaul (Fig. 1). At a second interconnection level, the CU connects to the distributed units (DUs) via the midhaul. Finally, the DU connects to the remote radio units (RRU) via the fronthaul. In this paper, we focus our attention on the fronthaul link and make use of an intra-RF split (functional split option 9) by distributing the RF signal between DU and RRU via Analog Radio-over-Fiber (ARoF). Often, ARoF schemes distributing mmWave signals are based on IF-over-Fiber (IFoF) [5]-[7], where a lower-frequency signal is distributed from the DU to the RRU and upconversion is required at the RRU before passing the signal to the wireless channel. This approach requires the distribution of a synchronous carrier, which is used to generate a local oscillator signal in the RRU. Furthermore, this heavily increases the complexity, since up- and downconversion for down- and uplink, respectively, are required at the RRU. 


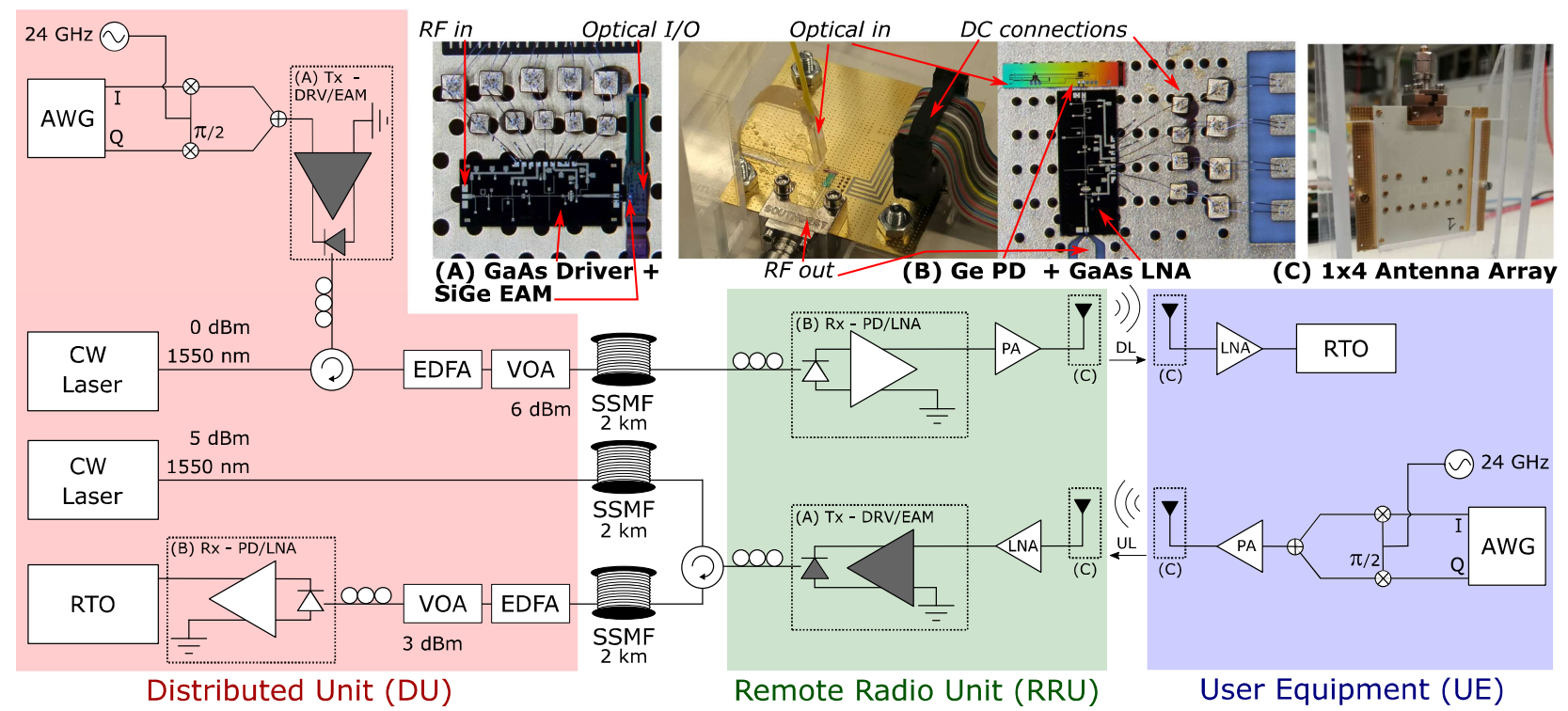

Fig. 2. Block diagram and experimental setup for bidirectional $28 \mathrm{GHz}$ RFoF link.

In this work, we propose an RF-over-Fiber (RFoF) implementation where the mmWave signal is directly distributed over the optical link. Therefore, the RRU only needs to perform conversion between the optical and RF domain, followed by an amplification to overcome the wireless path loss. The two main drawbacks of RFoF over IFoF are the need for highspeed electro-optic components and the presence of chromatic dispersion fading [8], [9]. The former is dealt with by using the high-speed silicon photonics platform ISIPP50G [10]. The latter can be countered by making use of optical single sideband (OSSB) transmission schemes [8], [11]. To reduce the complexity of the RRUs even further, laser-less operation of the uplink path is desired [12]-[17]. Reflective electro-absorption modulators (EAMs), with compact footprint, are devised to implement such a laser-less RRU. In contrast to the broadband electro-optic transmitters and receivers used in typical RoF links, a dedicated EAM-driver and photoreceiver were devised for optimal performance in the $28 \mathrm{GHz}$ band, using a combination of GaAs pHEMT electronics and silicon photonics.

This paper is an invited extension of our post-deadline work presented at OFC 2020 [18]. The proposed RFoF system features low-complexity, low-cost and easy-to-install RRUs, which is highly desired in centralized networks and distributed antenna systems (DAS). In Section II, the measurement setup is discussed together with a more detailed overview of the main building blocks. Subsequently, in Section III, the fiber-wireless link experiments are covered, demonstrating up to $12 \mathrm{~Gb} / \mathrm{s}$ transmission over $2 \mathrm{~km}$ standard single mode fiber (SSMF) and over $7 \mathrm{~Gb} / \mathrm{s}$ down- and uplink over a $2 \mathrm{~km}$ fiber and $5 \mathrm{~m}$ wireless link. For these experiments, the uplink operation involves two fibers to, on the one hand, distribute the unmodulated carrier and, on the other hand, send back the modulated uplink data. The effects of sharing the fiber for laser distribution and uplink transmission are described in Section IV. Finally, the results are compared with the state-of-the-art in Section V and a conclusion is provided in Section VI.

\section{EXPERIMENTAL SETUP}

The setup, implemented for the fiber-wireless experiments discussed in Section III, is depicted in Fig. 2. Up- and downlink are tested separately due to the Time Division Duplexing (TDD) scheme adopted in the targeted $5 \mathrm{G} \mathrm{New}$ Radio channels nr257/258 ranging from 24.25 to $29.5 \mathrm{GHz}$ [19]. The downlink path consists of an arbitrary waveform generator (AWG) that produces the desired I/Q samples, which are subsequently sent through an I/Q-mixer to construct the RF signal. This RF signal is fed to the narrowband driver (Section II-B) that drives a reflective electro-absorption modulator (EAM). The reflective EAM modulates a continuous wave $(\mathrm{CW}) 1550 \mathrm{~nm}$ laser source and the modulated light is separated from the unmodulated light using an optical circulator. An erbium doped fiber amplifier (EDFA) - variable optical attenuator (VOA) pair is used to set the optical power injected in the RoF link. At the RRU, the signal is converted back to the RF domain and amplified by a narrowband photoreceiver (Section II-A). The total power consumption of this narrowband $\mathrm{GaAs} / \mathrm{SiGe}$ chipset (driver and receiver) is $427 \mathrm{~mW}$. A commercial power amplifier (Analog Devices HMC943) is used to further boost the signal such that it is sufficiently strong for the wireless link. The transmitting and receiving antennas use beamforming to enhance the link budget even more and are discussed in Section II-C. When the signal arrives at the user equipment (UE) antenna array, the signal is first amplified by a commercial low-noise amplifier (LNA, Analog Devices HMC1040) and subsequently monitored by a real-time oscilloscope (RTO, Keysight DSA-Z634 A). The captured data is demodulated offline in Matlab.

The uplink path is similar. First, the RF signal is generated using an I/Q mixer to upconvert the IF signal generated by the AWG to the designated communication band. Next, the wireless channel is traversed and the signal arriving at the RRU is first amplified using a commercial LNA before being fed to the reflective narrowband transmitter. The unmodulated laser is modulated in the reflective EAM and separated from the $1550 \mathrm{~nm} \mathrm{CW}$ tone 


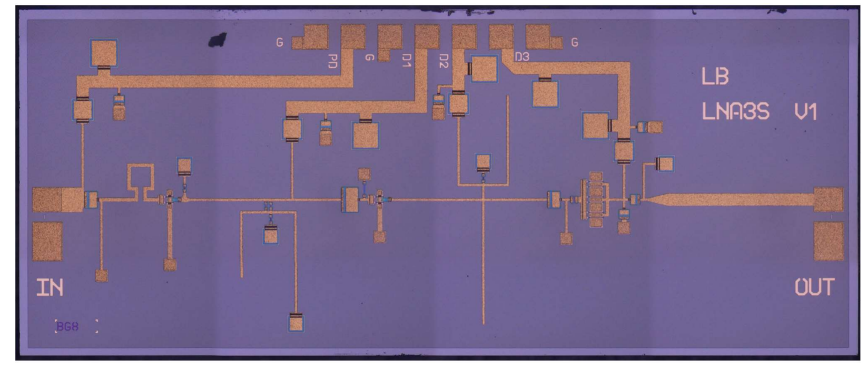

Fig. 3. Microscope image of the three-stage mmWave LNA

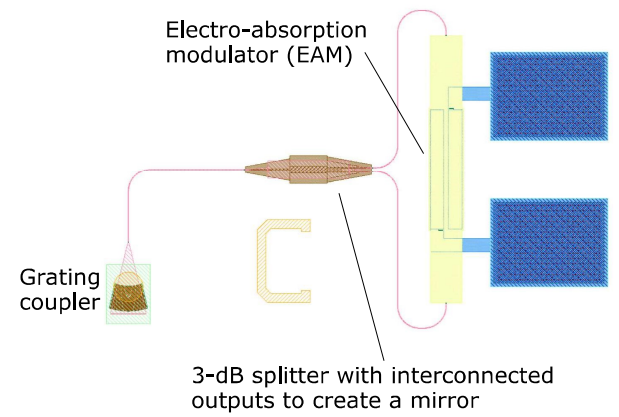

Fig. 4. Layout of the reflective EAM.

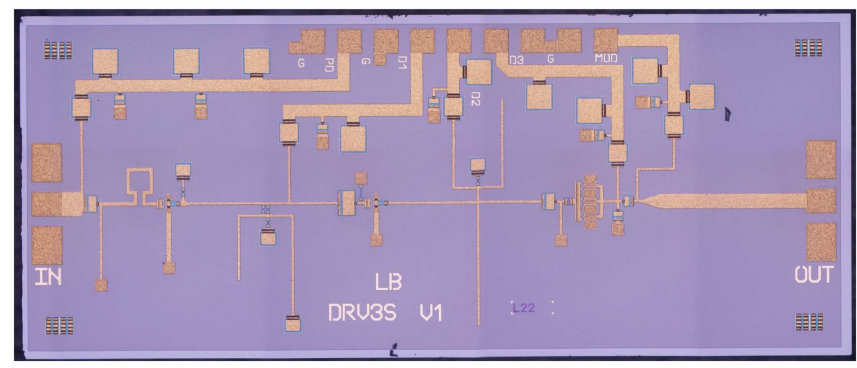

Fig. 5. Microscope image of the three-stage mmWave driver described extensively in [20]. It comprises a silicon waveguide coupled Ge-on-Si photodetector (PD) and a co-designed GaAs LNA. The receiver provides $24 \mathrm{~dB}$ gain, corresponding to $224 \mathrm{~V} / \mathrm{W}$ external conversion gain, over a $3-\mathrm{dB}$ bandwidth between 23.5 and $31.5 \mathrm{GHz}$. Furthermore, it offers a low noise figure of $2.12 \mathrm{~dB}$ at $28 \mathrm{GHz}$ and an output-referred third-order intercept point of $26.5 \mathrm{dBm}$ with a power consumption of $303 \mathrm{~mW}$. Consequently, the spurious free dynamic range (SFDR) of the photoreceiver is $116.3 \mathrm{~dB}^{-\mathrm{Hz}^{2 / 3}}$.

The resonant LNA is shown in Fig. 3 and consists of 3 amplification stages that can be biased independently. All three of these stages use common-source amplifiers where the first two include source degeneration to bring optimal source impedances for noise and gain matching closer together. The input of the amplifier is wirebonded to a Ge-on-Si PD and the input matching network includes an internal bias tee to counter inductance variations and source DC current generated by the PD. The output is AC coupled and is designed for a $50 \Omega$ load.

\section{B. Reflective EAM Driver}

The RFoF transmitter used for these experiments comprises a reflective EAM and a co-designed GaAs narrowband driver. The reflective EAM is shown in Fig. 4, where it is seen that integrated reflective operation is obtained by embedding the EAM in a loop constructed by interconnecting both outputs of a $3 \mathrm{~dB}$ splitter [21]. The devised narrowband driver is based on the core design of the LNA that is part of the photoreceiver. The first adjustment in the driver layout relative to the LNA layout is the addition of an internal bias tee at the output of the amplifier to allow for biasing of the modulator. Second, the source impedance changes from the PD-wirebond combination to $50 \Omega$ and the load is now a wirebond-EAM combination rather than the original $50 \Omega$ load expected in the optical receiver. Therefore, the input and output matching network were redesigned. The final layout is shown in Fig. 5. It is seen that, apart from the input, output and DC part, the design was kept identical to the LNA of the photoreceiver. Therefore, the driver offers a comparable small signal gain of $25.2 \mathrm{~dB}$ over a $3-\mathrm{dB}$ bandwidth between 24.4 and $29.5 \mathrm{GHz}$ with a noise figure of $2.07 \mathrm{~dB}$ at $28 \mathrm{GHz}$. Furthermore, the devised RFoF transmitter has an input-referred $1-\mathrm{dB}$ compression point of $-20 \mathrm{dBm}$ and consumes $124 \mathrm{~mW}$. Consequently, the SFDR of the RFoF transmitter is $107.7 \mathrm{~dB} . \mathrm{Hz}^{2 / 3}$ and will put an upper limit to the dynamic range of the RFoF link.

\section{C. $1 \times 4$ Corporate-Fed Antenna Array}

For the experiments discussed in this paper, a $1 \times 4$ uniform linear antenna array operating in the $28 \mathrm{GHz}$ band was deployed both as transmitting and receiving antenna. Both the backside and frontside are shown in Fig. 6, where the front side is the radiating side and the backside includes the feeding network. The individual antenna elements are air-filled cavity-backed patch antennas [22] and the 4-element antenna array is fed using a Wilkinson power-divider (WPD) distribution network. The antenna array provides fixed beamforming gain in the broadside direction. This increases the power received at the receive antenna and, therefore, boosts the sensitivity of the link. Furthermore, it reduces multipath fading effects, making the transmission over the wireless channel more robust. The WPD-fed antenna array exhibits a $-10 \mathrm{~dB}$ impedance bandwidth between $22.5 \mathrm{GHz}$ and $30.7 \mathrm{GHz}$, clearly covering both $5 \mathrm{G}$ new radio channels nr257/258 [19]. Furthermore, the antenna array offers a peak in-band gain of about $10 \mathrm{dBi}$ in the broadside direction, which corresponds to a beamforming gain of approximately $3.6 \mathrm{~dB}$ over the single antenna element. 


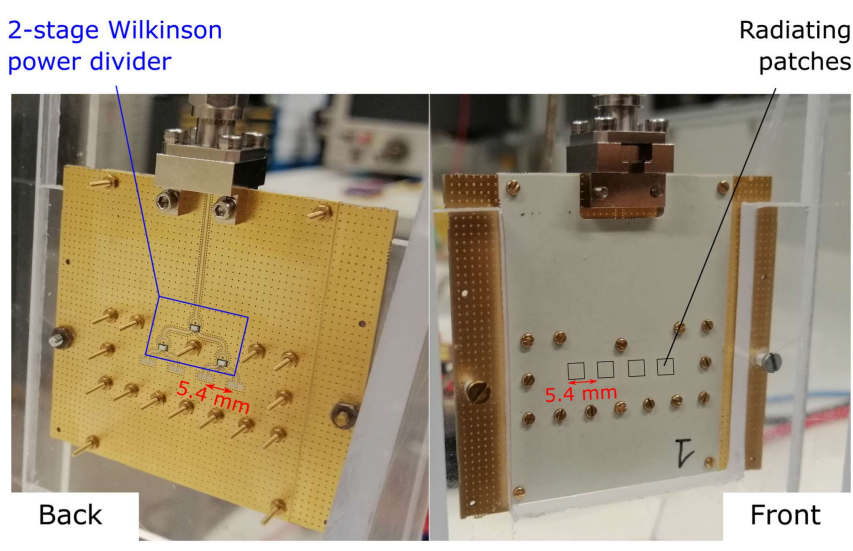

Fig. 6. Wilkinson-power-divider-fed $1 \times 4$ uniform linear antenna array covering the $\mathrm{nr} 257 / \mathrm{nr} 258$ bands $(24.25-29.5 \mathrm{GHz})$.

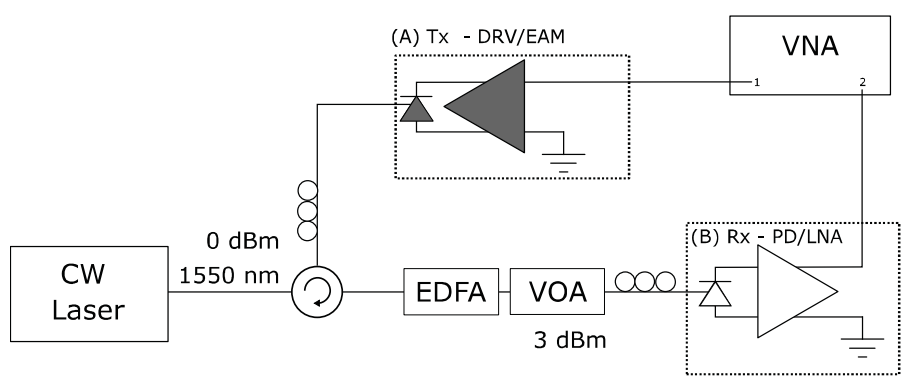

Fig. 7. Setup used to characterize the $\left|S_{21}\right|$ of the RFoF link.

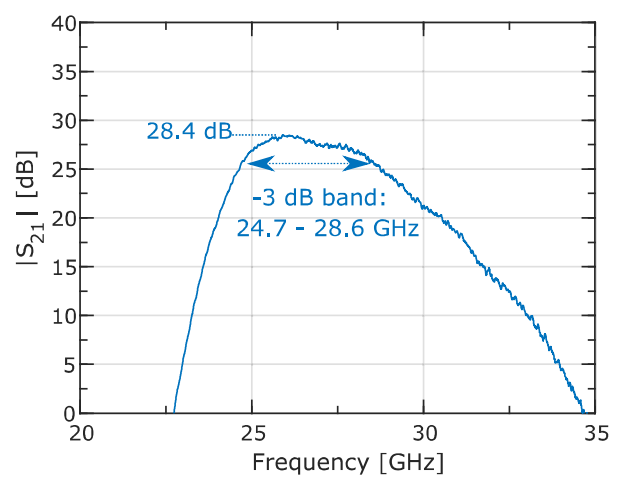

Fig. 8. $\left|S_{21}\right|$ of the narrowband RFoF link.

\section{RESULTS AND DISCUSSION}

The data transmission experiments are described in this section. First, the transfer function of the RFoF link (Fig. 7) is characterised using a Vector Network Analyzer (VNA). The resulting small-signal transmission $\left|S_{21}\right|$ is shown in Fig. 8. The 3-dB passband spans from 24.7 to $28.6 \mathrm{GHz}$, while the maximum transmission gain is $28.4 \mathrm{~dB}$ when $3 \mathrm{dBm}$ optical power is fed to the photoreceiver. Second, the fiber-wireless downlink is tested using single-carrier data transmission. Due to multi-path effects in the wireless channel, large wireless distances show strong fading dips. Hence, in the final part of this section, orthogonal frequency domain multiplexing (OFDM) is applied to transmit the data from the RRU to the UE. The up- and downlink path are tested separately because of the envisioned TDD [19].
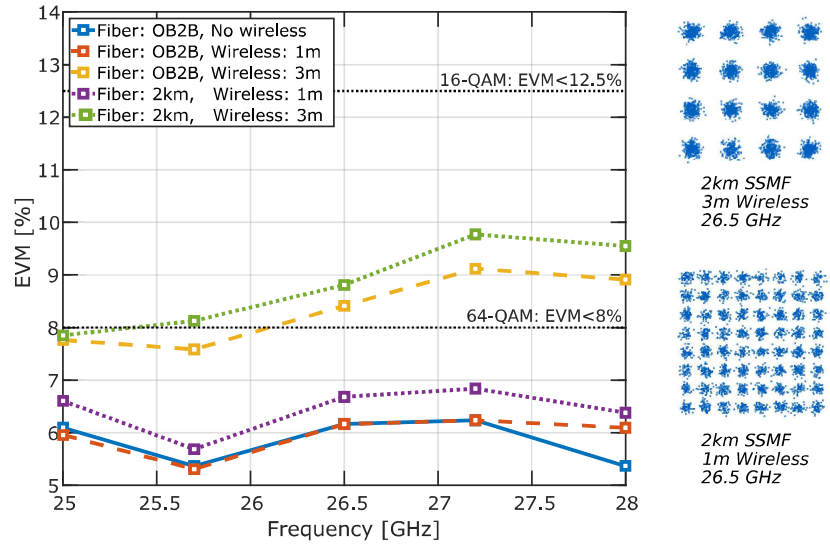

Fig. 9. Measured EVM per channel for 5 x 400 MBaud multiband singlecarrier RFoF-wireless downlink

\section{A. Single-Carrier}

To explore the maximum data capacity of the implemented fiber-wireless link, multi-channel downlink communication was evaluated. Five $400 \mathrm{MBd}$ channels were transmitted simultaneously over the fiber-wireless link. These channels are centered around 25.0, 25.7, 26.5, 27.2 and $28 \mathrm{GHz}$. They are constructed using a roll-off factor of 0.2 . The resulting error vector magnitude (EVM), normalized to the average power, is depicted in Fig. 9. First, the optical back-to-back (OB2B) performance was measured without wireless path, resulting in EVM values $(\mathrm{EVM}<6.2 \%$ ) well below the $8 \%$ criterion set by the $3 \mathrm{GPP}$ specifications for 64-QAM transmission [23]. When $1 \mathrm{~m}$ of wireless distance is added between the RRU and the UE, the EVM degradation is negligible. The penalty caused by the addition of $2 \mathrm{~km}$ SSMF in between the DU and the RRU, mimicking a distributed wireless infrastructure, results in a minor EVM degradation (EVM $<6.8 \%$ ) but there is still sufficient margin relative to the $8 \%$ EVM specification. Consequently, when limiting the fiber distance to $2 \mathrm{~km}$ and the wireless distance to $1 \mathrm{~m}$, the devised mmWave-over-Fiber link provides a data capacity of at least $12 \mathrm{Gbps}$. By increasing the wireless distance to $3 \mathrm{~m}$, the $8 \%$ criterion is no longer met and now 16-QAM is the largest constellation size that can be adopted to stay within specifications since the target EVM for 16-QAM is $12.5 \%$ [23].

\section{B. Orthogonal Frequency Division Multiplexing}

When larger wireless distances are desired, OFDM offers better performance than single-carrier data modulation, since it is more robust to multipath fading [24]. The main drawback of the OFDM data transmission is the increase in peak-to-averagepower ratio (PAPR), requiring higher linearity $\mathrm{E} / \mathrm{O}$ and $\mathrm{O} / \mathrm{E}$ converters and its associated drivers and amplifiers. The signal parameters of the OFDM data can be found in Table I. The resulting data rate per channel is $2.34 \mathrm{~Gb} / \mathrm{s}$ when 16-QAM signals are transmitted and increases to $3.51 \mathrm{~Gb} / \mathrm{s}$ per channel when the link has a sufficiently low EVM to allow for 64-QAM data communication. The down- and uplink signal quality are displayed in Fig. 10. First, it can be seen that the downlink and uplink performance are comparable and that there is an EVM 
TABLE I

OFDM SIGNAL PARAMETERS

\begin{tabular}{|r|l|}
\hline Bandwidth & $800 \mathrm{MHz}$ \\
Number of subcarriers & 512 \\
Number of pilots & 16 \\
Number of DC, null subcarriers & 28 \\
Cyclic prefix (CP) size & $1 / 4$ \\
Peak-to-average-ratio & $10.8 \mathrm{~dB}$ \\
Data rate per channel (16-QAM) & $2.34 \mathrm{~Gb} / \mathrm{s}$ \\
\hline
\end{tabular}

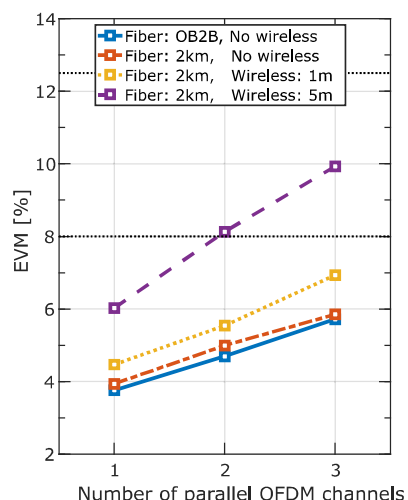

(a) Downlink

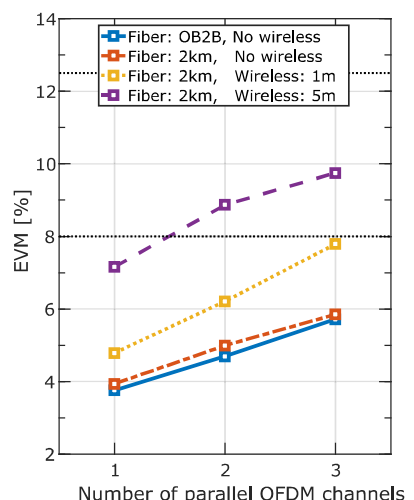

(b) Uplink
Fig. 10. Measured EVM in RFoF-wireless link.

degradation when multiple channels are transmitted simultaneously. The introduction of $2 \mathrm{~km} \mathrm{SSMF}$ between DU and RRU has limited impact and the EVM stays below the 64-QAM boundary for a $1 \mathrm{~m}$ wireless channel. Up to $5 \mathrm{~m}$ wireless link distance was tested with this setup, resulting in EVM values below 10\%. An aggregated capacity of $7.02 \mathrm{~Gb} / \mathrm{s}$ was achieved over $2 \mathrm{~km} \mathrm{SSMF}$ and $5 \mathrm{~m}$ wireless distance for both downlink and uplink with an EVM that meets the 3GPP specification. When the wireless distance is limited to $1 \mathrm{~m}$, the EVM values demonstrate that up to $10.53 \mathrm{~Gb} / \mathrm{s}$ can be reached for the devised fiber-wireless link.

\section{REFLECTIVE MODULATOR OPERATION}

In a small-cell architecture, it would be advantageous if the laser could be omitted from the RRU to reduce the cost and power consumption [25], [26]. The centralization of the light generation for the uplink path allows for sharing the laser between multiple cells and relaxes the stringent temperature control requirements at the RRUs. In the previous experiments, the reflective transmitter was converted to a regular transmitter by using an optical circulator and by using separate fibers to distribute the uplink CW laser light to the RRU and to send the modulated light back to the DU (setup A in Fig. 11).

\section{A. Rayleigh Backscattering}

This section discusses the effect of sharing fiber for laser distribution and uplink communication and, thereby, introducing true reflective operation (setup B in Fig. 11). In Fig. 12, the EVM degradation due to reflective operation is studied when transmitting single-carrier, single-channel 16-QAM signals with a symbol rate of 100 MBaud. In the original setup (scenario A), the signal passes in one direction over the fiber. However, when true reflective operation is adopted (scenario B), Rayleigh scattering occurs along the fiber. This results in strong signal degradation, especially at large fiber lengths. The unmodulated light that is sent to the reflective EAM undergoes distributed reflections while passing along the fiber and this reflected $\mathrm{CW}$ tone interferes with the carrier of the modulated light traveling back from the reflective EAM over that same fiber [27]. After $2 \mathrm{~km}$ fiber, the EVM increases from $2.48 \%$ to $7.33 \%$ due to backscattering and after $10 \mathrm{~km}$ fiber the EVM increases from $2.51 \%$ to $23.44 \%$ when adopting true reflective operation. Furthermore, it is clear that a $4.5 \mathrm{~km}$-long fiber also results in poor signal quality. This is due to chromatic dispersion, as can be calculated from [9]. In Fig. 13, the received constellation diagrams in setup A and B are shown for optical back-to-back, $4.5 \mathrm{~km}$ SSMF (corresponding with the dispersion notch) and $10 \mathrm{~km}$ SSMF. It is clear that both setups are identical for OB2B and cannot be used at the dispersion distance of $4.5 \mathrm{~km}$. At $10 \mathrm{~km}$, it can be seen that Rayleigh backscattering introduces strong parasitic intensity modulation, indicating that setup B can only be used for small DU-RRU distances.

\section{B. Edge Coupling}

Reducing the insertion loss of the modulator will help to lower the effect of the Rayleigh scattering, since the relative level between modulated light and scattered CW laser will be larger. The transmitter used in these experiments relies on grating couplers to couple the light in and out of the photonic IC. While this eases testing, it also results in a higher coupling loss than what is achievable with edge coupling [28]. Hence, migration from a grating to an edge coupling technique will help to reduce the penalty due to Rayleigh scattering noise.

\section{Coherent Detection}

To truly solve the issue, we should remove the carrier of the signal received at the DU. This can, for example, be achieved by locking a micro-ring resonator to the carrier wavelength. However, we opted to use a tunable optical bandpass filter (OTF-350). This approach not only removes the carrier but also one of the sidebands, resulting in a carrier-suppressed optical single sideband (CS-OSSB) detection scheme that simultaneously solves the Rayleigh backscattering as well as the chromatic-dispersion-induced fading dips. The proposed uplink configuration is shown in Fig. 11 as setup $\mathrm{C}$ and combines true reflective operation of the RRU with coherent detection of the uplink at the DU. The 50/50 blocks in this setup are $3 \mathrm{~dB}$ splitters/combiners. The output constellation diagrams in Fig. 14 show three distinct rings instead of the typical ideal 16-QAM constellation visible in the OB2B measurements in Fig. 13. This is due to the coherent detection scheme that was implemented with a laser with $400 \mathrm{kHz}$ linewidth. Frequency tracking and/or a smaller linewidth laser is required for coherent detection. However, it is already clear that both Rayleigh backscattering and chromatic dispersion degradation can be solved by using setup $\mathrm{C}$, since we can clearly discriminate the three amplitude levels of the 16-QAM symbols. Using offline processing to implement a 

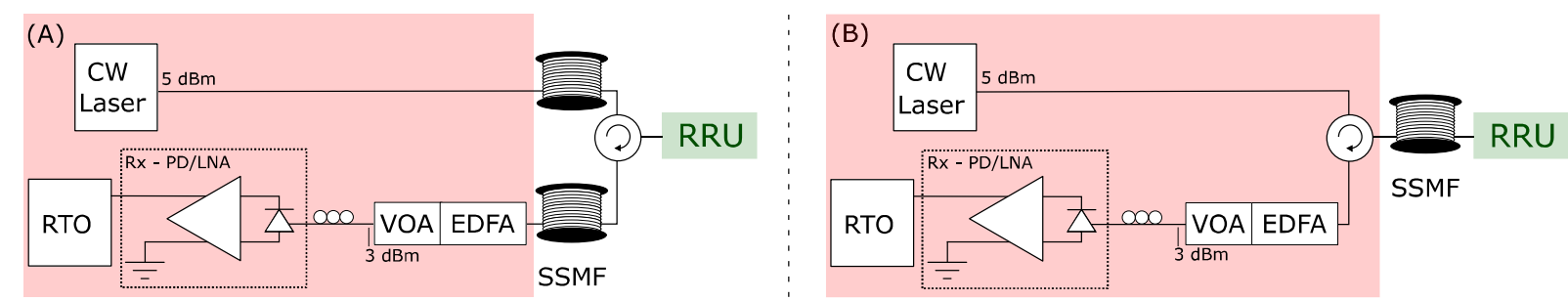

(C)

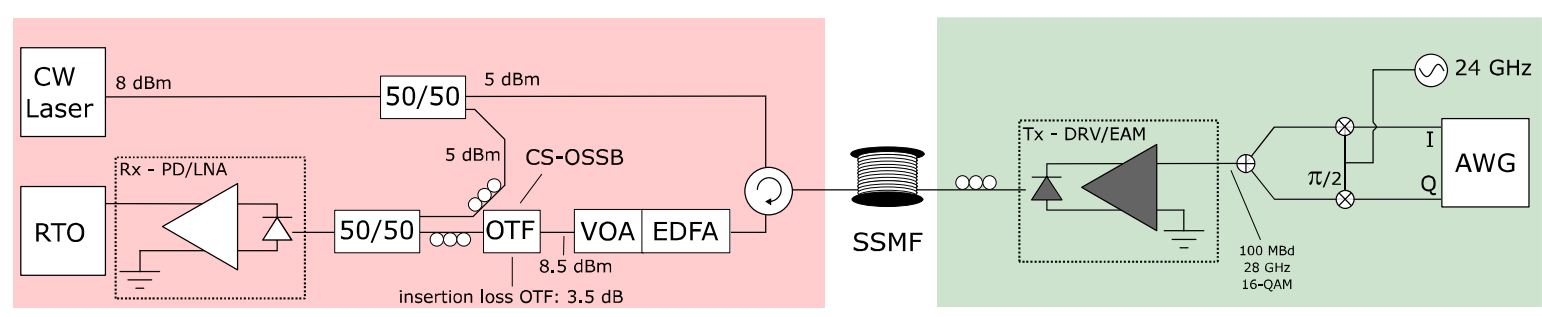

Fig. 11. Uplink implementations: (A) direct detection with separate fiber for carrier distribution and modulated uplink signal; (B) direct detection with shared fiber; (C) coherent detection with shared fiber.

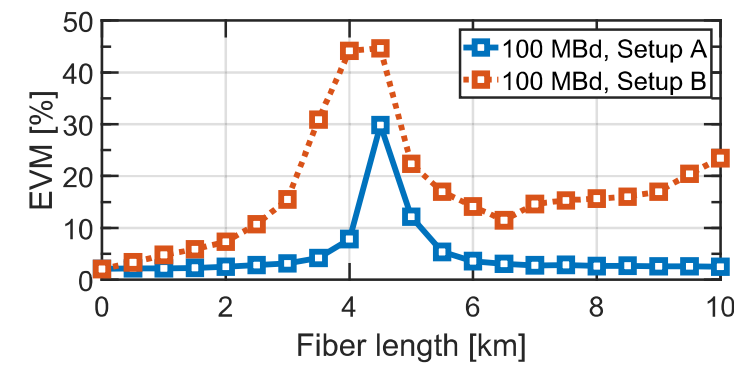

Fig. 12. EVM degradation due to true reflective operation using direct detection.

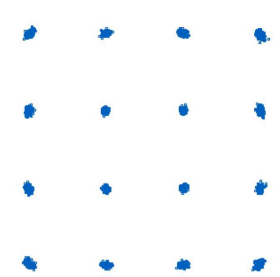

(a) Setup A, OB2B

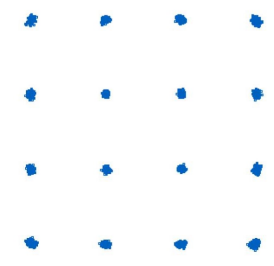

(d) Setup B, OB2B

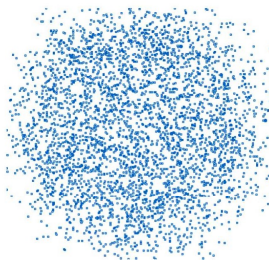

(b) Setup A, $4.5 \mathrm{~km}$

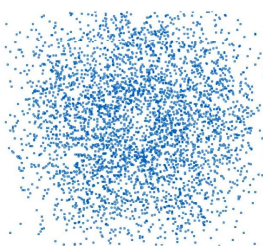

(e) Setup B, $4.5 \mathrm{~km}$

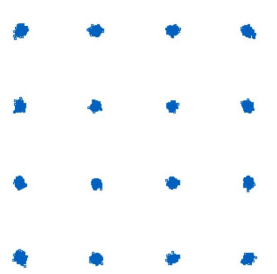

(c) Setup A, $10 \mathrm{~km}$

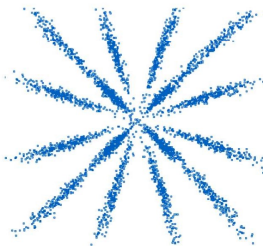

(f) Setup B, $10 \mathrm{~km}$
Fig. 13. Received signal when transmitting a 100 MBaud, 16-QAM modulated data signal and using direct detection.

digital Costas phase-locked loop at the receiver results in the bottom constellation diagrams shown in Fig. 14. Therefore, it can be concluded that this setup can be used to implement true reflective RRUs with larger DU-RRU separation and that it simultaneously

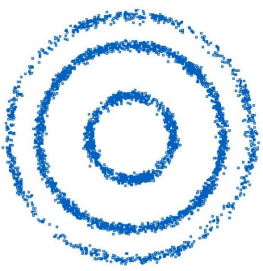

(a) Setup C, OB2B

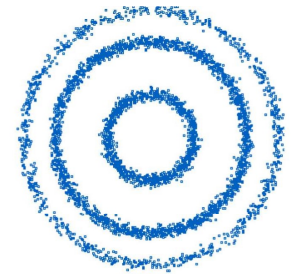

(b) Setup C, $4.5 \mathrm{~km}$

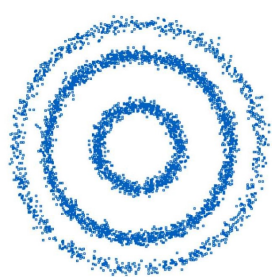

(c) Setup C, $10 \mathrm{~km}$

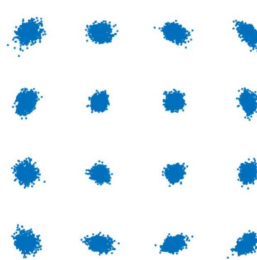

(d) Setup C, FT, OB2B, $\mathrm{EVM}=5.78 \%$

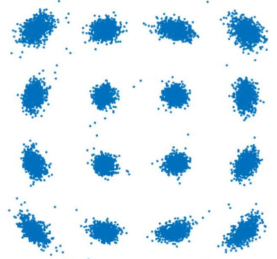

(e) Setup C, FT, $4.5 \mathrm{~km}$, $\mathrm{EVM}=8.21 \%$

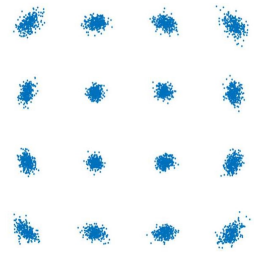

(f) Setup C, FT, $10 \mathrm{~km}$ $\mathrm{EVM}=6.19 \%$
Fig. 14. Received signal when transmitting a 100 MBaud, 16-QAM modulated data signal and using coherent detection (FT: frequency tracking).

solves the dispersion challenge inherent to high carrier frequency RFoF implementation schemes. While the implemented system is adequate as a proof-of-principle, practical implementations should include polarization tracking. Typical coherent links [29], [30] implement this via polarization beam splitters after which two coherent receivers detect the two polarizations. These two outputs are then combined and digital signal processing (DSP) is used to reconstruct the transmitted uplink signal. Alternatively, one can use a polarization transformer with feedback loop to stabilize the polarization [31].

\section{Proposed Architecture}

Based on the findings in section IV-C, it is now possible to devise a single fiber architecture. The proposed full duplex link is shown in Fig. 15 and can be used independent of the required 
TABLE II

StATE OF THE ART FIBER-WirEless LinK EXPERIMENTS (DL: DOWNLINK; UL: UPLINK; SC: SingLE-CARRIER; SPC: SUPERPOSITION CODE; UFOFDM:

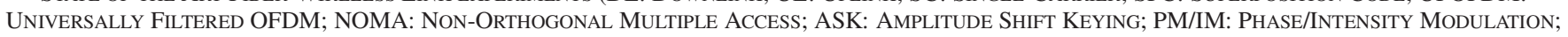
FDD: FREQuENCY DIVISION DuPLEXING; SDOF: SigMA-DELTA-OVER-FIBER; MMF: Multimode FIBER)

\begin{tabular}{|c|c|c|c|c|c|c|c|c|c|c|}
\hline & Bitrate & Signal & $\begin{array}{c}\text { RF } \\
\text { Band }\end{array}$ & Fiber & Wireless & $\begin{array}{l}\text { RoF } \\
\text { type }\end{array}$ & DL & $\mathbf{U L}$ & $\begin{array}{c}\text { Laser } \\
\text { at RRU }\end{array}$ & Extra info \\
\hline [18] & $8 \mathrm{~Gb} / \mathrm{s}$ & SC-16QAM & $28 \mathrm{GHz}$ & $2 \mathrm{~km}$ & $3 \mathrm{~m}$ & RFoF & $\checkmark$ & & & \\
\hline [18] & $12 \mathrm{~Gb} / \mathrm{s}$ & SC-64QAM & $28 \mathrm{GHz}$ & $2 \mathrm{~km}$ & $1 \mathrm{~m}$ & RFoF & $\checkmark$ & & & \\
\hline [18] & $7.02 \mathrm{~Gb} / \mathrm{s}(\mathrm{DL} / \mathrm{UL})$ & OFDM-16QAM & $28 \mathrm{GHz}$ & $2 \mathrm{~km}$ & $5 \mathrm{~m}$ & RFoF & $\checkmark$ & $\checkmark$ & No & \\
\hline [18] & $10.53 \mathrm{~Gb} / \mathrm{s}(\mathrm{DL} / \mathrm{UL})$ & OFDM-64QAM & $28 \mathrm{GHz}$ & $2 \mathrm{~km}$ & $1 \mathrm{~m}$ & RFoF & $\checkmark$ & $\checkmark$ & No & \\
\hline [33] & $3.39 \mathrm{~Gb} / \mathrm{s}(\mathrm{DL})+498 \mathrm{Mb} / \mathrm{s}(\mathrm{UL})$ & OFDM-64QAM & $28 \mathrm{GHz}$ & $2 \mathrm{~km}$ & $3 \mathrm{~m}$ & IFoF & $\checkmark$ & $\checkmark$ & Yes & $4 \times 4$ MIMO, TDD \\
\hline [33] & $870 \mathrm{Mb} / \mathrm{s}(\mathrm{DL})+250 \mathrm{Mb} / \mathrm{s}(\mathrm{UL})$ & OFDM-64QAM & $28 \mathrm{GHz}$ & $2 \mathrm{~km}$ & $3 \mathrm{~m}$ & IFoF & $\checkmark$ & $\checkmark$ & Yes & TDD \\
\hline [34] & $4.56 \mathrm{~Gb} / \mathrm{s}$ & UFOFDM-64QAM & $28 \mathrm{GHz}$ & $25 \mathrm{~km}$ & $0.1 \mathrm{~m}$ & RFoF & $\checkmark$ & & & \\
\hline [35] & $12 \mathrm{~Gb} / \mathrm{s}$ & OFDM-16QAM & $28 \mathrm{GHz}$ & $50 \mathrm{~km}$ & $3 \mathrm{~m}$ & RFoF & $\checkmark$ & & & \\
\hline [4] & $1.37 \mathrm{~Gb} / \mathrm{s}$ & OFDM-64QAM & $28 \mathrm{GHz}$ & $100 \mathrm{~m}$ & $1.5 \mathrm{~m}$ & SDoF & $\checkmark$ & & & MMF \\
\hline [36] & $8 \mathrm{~Gb} / \mathrm{s}$ & SPC-NOMA & $60 \mathrm{GHz}$ & $3 \mathrm{~km}$ & $2.5 \mathrm{~m}$ & RFoF & $\checkmark$ & & & 2 users \\
\hline$[6]$ & $24 \mathrm{~Gb} / \mathrm{s}(\mathrm{DL} / \mathrm{UL})$ & SC-16QAM & $60 \mathrm{GHz}$ & $7 \mathrm{~km}$ & $5 \mathrm{~m}$ & IFoF & $\checkmark$ & $\checkmark$ & Yes & $\mathrm{DFB}+\mathrm{MZM}$ \\
\hline [6] & $16 \mathrm{~Gb} / \mathrm{s}$ & SC-16QAM & $60 \mathrm{GHz}$ & $7 \mathrm{~km}$ & $5 \mathrm{~m}$ & IFoF & $\checkmark$ & & & EML \\
\hline [6] & $12 \mathrm{~Gb} / \mathrm{s}(\mathrm{DL} / \mathrm{UL})$ & SC-QPSK & $60 \mathrm{GHz}$ & $7 \mathrm{~km}$ & $5 \mathrm{~m}$ & $\mathrm{IFoF}$ & $\checkmark$ & $\checkmark$ & Yes & EML \\
\hline [37] & $600 \mathrm{Mb} / \mathrm{s}$ & QPSK & $60 \mathrm{GHz}$ & $1 \mathrm{~km}$ & $1 \mathrm{~m}$ & IFoF & & $\checkmark$ & Yes & Beamsteering: $15^{\circ}$ \\
\hline [38] & $24.08 \mathrm{~Gb} / \mathrm{s}$ & OFDM-16QAM & $60 \mathrm{GHz}$ & $20 \mathrm{~km}$ & $4 \mathrm{~m}$ & RFoF & $\checkmark$ & & & \\
\hline [38] & $24.08 \mathrm{~Gb} / \mathrm{s}$ & OFDM-16QAM & $91 \mathrm{GHz}$ & $20 \mathrm{~km}$ & $3 \mathrm{~m}$ & RFoF & $\checkmark$ & & & \\
\hline [15] & $2.5 \mathrm{~Gb} / \mathrm{s}(\mathrm{DL})+2.5 \mathrm{~Gb} / \mathrm{s}(\mathrm{UL})$ & ASK & $40 \mathrm{GHz}$ & $40 \mathrm{~km}$ & N/A & RFoF & $\checkmark$ & $\checkmark$ & No & FDD \\
\hline [16] & $850 \mathrm{Mb} / \mathrm{s}(\mathrm{DL})+850 \mathrm{Mb} / \mathrm{s}(\mathrm{UL})$ & PM(DL)-IM(UL) & $5.25 \mathrm{GHz}$ & $25 \mathrm{~km}$ & N/A & RFoF & $\checkmark$ & $\checkmark$ & No & Reflective SOA \\
\hline [17] & $1 \mathrm{~Gb} / \mathrm{s}(\mathrm{DL})+1 \mathrm{~Gb} / \mathrm{s}(\mathrm{UL})$ & ASK & $60 \mathrm{GHz}$ & $10 \mathrm{~km}$ & N/A & RFoF & $\checkmark$ & $\checkmark$ & No & FDD \\
\hline
\end{tabular}

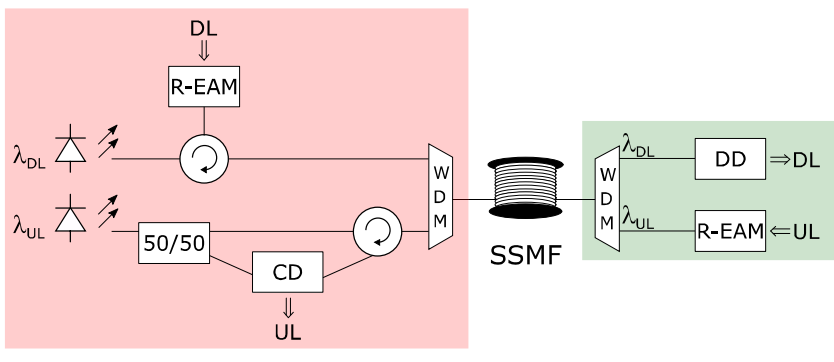

Fig. 15. Proposed single-fiber architecture (R-EAM: reflective EAM; WDM: wavelength division multiplexer; $\mathrm{CD}$ : coherent detection; DD: direct detection).

duplexing strategy. In this setup, the down- and uplink are implemented using two separate wavelengths. Meanwhile, the laser distribution and back-transmission of the modulated uplink data can occur over the same fiber by using coherent detection of the uplink to avoid Rayleigh backscattering signal degradation. The proposed scheme requires no circulators at the RRU and all optical functionality of the RRU (WDM, photodetector, and EAM) can be implemented on a single silicon photonics IC. This architecture can be extended to a network serving multiple RRUs. Different interconnection strategies can be followed to serve multiple access points. One can for example deploy a star, multilevel-star, or ring configuration [32] where the most optimal solution depends on the network requirements. The most straightforward technique is the star topology where every RRU is addressed by a point-to-point link, however, this requires the maximum amount of fiber since each RRU is addressed by a dedicated fiber. In this scenario, only two unique wavelengths are required, one for downlink and one for uplink communication, no matter the number of RRUs. In the multilevel-star approach, the number of required wavelengths is increased to reduce the total fiber cost. Assuming that the maximum number of RRUs served by a single fiber is $\mathrm{N}$, then the multilevel-star topology will require $2 \times \mathrm{N}$ unique wavelengths and the data is routed to the intended RRU by WDM routing.

\section{State of the ART}

Table II shows a state of the art overview of fiber-wireless link experiments. A great interest is seen for the licensed $28 \mathrm{GHz}$ and unlicensed $60 \mathrm{GHz}$ band. It is shown that our setup provides state of the art performance regarding achieved bitrate, fiber distance and wireless distance. The fiber length is sufficient for typical applications and can be increased significantly by using an optical single sideband scheme to avoid fading dips resulting from fiber dispersion. The link setup discussed in this paper not only shows good performance for downlink but also for the uplink communication. While this uplink is typically implemented by including a laser at the RRU, it is not ideal with respect to cost, size, etc. Therefore, we propose to use centralised laser generation. Three references that show RoF implementations where the laser light is omitted from the RRU but without wireless path were added to table II, being [15], [16] and [17]. In [16], a single fiber solution is devised using reflective semiconductor optical amplifier (SOA) to modulate the uplink light. Due to amplification in the SOA, Rayleigh issues are relaxed. In [15] and [17], a two-fiber solution is adopted, hence the backscattered carrier does not couple back to the receiver for the uplink path.

\section{CONCLUSION}

We have demonstrated a very low complexity narrowband GaAs electronics/Si photonics transceiver for scalable RFoF architectures. The chipset consumes $427 \mathrm{~mW}$, introduces a link gain of $28.4 \mathrm{~dB}$ - with $3 \mathrm{dBm}$ optical power - and supports a link bandwidth from 24.7 to $28.6 \mathrm{GHz}$. Furthermore, laser-free remote radio unit operation is enabled using RFoF transmitters with reflective EAMs, which reduces the complexity of the remote radio units even further. With this transceiver, over $7 \mathrm{~Gb} / \mathrm{s}$ down- and uplink were demonstrated for a $2 \mathrm{~km}$ fiber, $5 \mathrm{~m}$ wireless mmWave link. In the final part of this paper, the focus shifted to enable true reflective operation over longer fiber lengths. Direct detection schemes suffer from Rayleigh 
backscattering, making the reflective operation in combination with direct detection infeasible for larger DU-RRU distances. Edge coupling can be used to relax the Rayleigh degradation to some extent and coherent detection, in combination with frequency tracking, can be used to get rid of the Rayleigh backscattering altogether. Furthermore, when CS-OSSB is used in combination with coherent detection, it not only solves the Rayleigh backscattering but also overcomes chromatic dispersion limitations inherent to high carrier frequency RFoF.

\section{REFERENCES}

[1] Ericsson, "Ericsson mobility report november 2019," Nov. 2019. [Online]. Available: https://www.ericsson.com/en/mobility-report/reports

[2] J. G. Andrews et al., "What will 5 G be?" IEEE J. Sel. Areas Commun., vol. 32 , no. 6 , pp. 1065-1082, Jun. 2014

[3] C. I, H. Li, J. Korhonen, J. Huang, and L. Han, "RAN revolution with NGFI (xhaul) for 5 G," J. Lightw. Technol., vol. 36, no. 2, pp. 541-550, Jan. 2018

[4] C.-Y. Wu et al., "Distributed antenna system using sigma-delta intermediate-frequency-over-fiber for frequency bands above $24 \mathrm{GHz}$," J. Lightw. Technol., vol. 38, no. 10, pp. 2765-2773, May 2020.

[5] J. Kim et al., "OTA enabled $147.4 \mathrm{~Gb} / \mathrm{s}$ eCPRI-equivalent-rate radiooverfiber link cooperating with mmWave-based Korea Telecom $5 \mathrm{G}$ mobile network for distributed antenna system," in Proc. Opt. Fiber Commun. Conf., 2019, Paper Th4C.5.

[6] N. Argyris et al., "A $5 \mathrm{G}$ mmWave fiber-wireless IFoF analog mobile fronthaul link with up to 24-Gb/s multiband wireless capacity," J. Lightw. Technol., vol. 37, no. 12, pp. 2883-2891, Jun. 2019.

[7] M. Sung, S. Cho, J. Kim, J. K. Lee, J. H. Lee, and H. S. Chung, "Demonstration of IFoF-based mobile fronthaul in $5 \mathrm{G}$ prototype with 28-GHz millimeter wave," J. Lightw. Technol., vol. 36, no. 2, pp. 601-609, Jan. 2018.

[8] C. Lim et al., "Fiber-wireless networks and subsystem technologies," $J$. Lightw. Technol., vol. 28, no. 4, pp. 390-405, Feb. 2010.

[9] U. Gliese, S. Norskov, and T. N. Nielsen, "Chromatic dispersion in fiberoptic microwave and millimeter-wave links," IEEE Trans. Microw. Theory Tech., vol. 44, no. 10, pp. 1716-1724, Oct. 1996.

[10] M. Pantouvaki et al., "Active components for $50 \mathrm{~Gb} / \mathrm{s}$ NRZ-OOK optical interconnects in a silicon photonics platform," J. Lightw. Technol., vol. 35, no. 4, pp. 631-638, Feb. 2017.

[11] T. Cseh et al., "Dispersion compensation in millimeter wave radio over multimode fiber systems," Microw. Opt. Technol. Lett., vol. 57, no. 1, pp. 204-207, Jan. 2015

[12] D. Wake et al., "Passive picocell: A new concept in wireless network infrastructure," Electron. Lett., vol. 33, no. 5, pp. 404-406, Feb. 1997.

[13] A. Stohr, K. Kitayama, and D. Jager, "Full-duplex fiber-optic RF subcarrier transmission using a dual-function modulator/photodetector," IEEE Trans. Microw. Theory Tech., vol. 47, no. 7, pp. 1338-1341, Jul. 1999.

[14] A. Nirmalathas, D. Novak, C. Lim, and R. B. Waterhouse, "Wavelength reuse in the WDM optical interface of a millimeter-wave fiber-wireless antenna base station," IEEE Trans. Microw. Theory Tech., vol. 49, no. 10, pp. 2006-2012, Oct. 2001.

[15] J. Yu, Z. Jia, T. Wang, and G. Chang, "A novel radio-over-fiber configuration using optical phase modulator to generate an optical mm-wave and centralized lightwave for uplink connection," IEEE Photon. Technol. Lett., vol. 19, no. 3, pp. 140-142, Feb. 2007.

[16] X. Yu, T. B. Gibbon, and I. T. Monroy, "Bidirectional radio-over-fiber system with phase-modulation downlink and RF oscillator-free uplink using a reflective SOA," IEEE Photon. Technol. Lett., vol. 20, no. 24, pp. 2180-2182, Dec. 2008.

[17] T. Taniguchi, N. Sakurai, K. Kumozaki, and T. Imai, "Full-duplex 1.0 Gbit/s data transmission over $60 \mathrm{GHz}$ radio-on-fiber access system based on the loop-back optical heterodyne technique," J. Lightw. Technol., vol. 26, no. 13, pp. 1765-1776, Jul. 2008.
[18] L. Bogaert et al., "SiPhotonics/GaAs 28-GHz transceiver for mmwaveover-fiber laser-less active antenna units," in Proc. Opt. Fiber Commun. Conf., 2020, Paper Th4A.5.

[19] 3GPP, "New frequency range for NR (24.25-29.5 GHz) - Release 15, v15.0.0," Tech. Rep. TR 38.815, Jul. 2018. [Online]. Available: https:// www.3gpp.org/dynareport/38-series.htm

[20] L. Bogaert et al., " $36 \mathrm{~Gb} / \mathrm{s}$ narrowband photoreceiver for mmwave analog radio-over-fiber," J. Lightw. Technol., vol. 38, no. 12, pp. 3289-3295, Jun. 2020 .

[21] F. Soltani et al., "Integrated silicon photonic reflective modulator for passive optical networks," in Proc. Conf. Lasers Electro-Opt., 2017, Paper JW2A.127.

[22] Q. Van den Brande, S. Lemey, J. Vanfleteren, and H. Rogier, "Highly efficient impulse-radio ultra-wideband cavity-backed slot antenna in stacked air-filled substrate integrated waveguide technology," IEEE Trans. Antennas Propag., vol. 66, no. 5, pp. 2199-2209, May 2018.

[23] 3GPP, "LTE; Evolved Universal Terrestrial Radio Access (E-UTRA); Base Station (BS) radio transmission and reception - Release 15 - v 15.3.0," Tech. Rep. TR 36.104, Jul. 2018. [Online]. Available: https://www.3gpp. org/dynareport/36-series.htm

[24] A. A. Zaidi et al., "Waveform and numerology to support $5 \mathrm{G}$ services and requirements," IEEE Commun. Mag., vol. 54, no. 11, pp. 90-98, Nov. 2016.

[25] Z. Tang and S. Pan, "A full-duplex radio-over-fiber link based on a dual-polarization mach-zehnder modulator," IEEE Photon. Technol. Lett., vol. 28 , no. 8, pp. 852-855, Apr. 2016

[26] Z. Jia et al., "A full-duplex radio-over-fiber system with $2.5 \mathrm{Gbit} / \mathrm{s}$ data symmetric delivery over $40 \mathrm{~km}$ SMF-28," in Proc. Eur. Conf. Opt. Commun., 2006, Paper Tu1.6.3.

[27] G. Talli et al., "Rayleigh noise mitigation in longreach hybrid DWDMTDM PONs," J. Opt. Netw., vol. 6, no. 6, pp. 765-776, Jun. 2007.

[28] R. Marchetti et al., "Coupling strategies for silicon photonics integrated chips," Photon. Res., vol. 7, no. 2, pp. 201-238, Feb. 2019.

[29] K. Kikuchi, "Fundamentals of coherent optical fiber communications," $J$. Lightw. Technol., vol. 34, no. 1, pp. 157-179, Jan. 2016.

[30] S. J. Savory, "Digital coherent optical receivers: Algorithms and subsystems," IEEE J. Sel. Top. Quantum Electron, vol. 16, no. 5, pp. 1164-1179, Sep./Oct. 2010.

[31] A. Hidayat et al., "High-speed endless optical polarization stabilization using calibrated waveplates and field-programmable gate array-based digital controller," Opt. Express, vol. 16, no. 23, pp. 18984-18991, Nov. 2008.

[32] J. Beas, G. Castanon, I. Aldaya, A. Aragon-Zavala, and G. Campuzano, "Millimeter-wave frequency radio over fiber systems: A survey," IEEE Commun. Surv. Tut., vol. 15, no. 4, pp. 1593-1619, Oct.-Dec. 2013.

[33] J. Kim et al., "MIMO-supporting radio-over-fiber system and its application in mmWave-based indoor $5 \mathrm{G}$ mobile network," J. Lightw. Technol., vol. 38, no. 1, pp. 101-111, Jan. 2020.

[34] E. Martin et al., " $28 \mathrm{GHz} 5 \mathrm{G}$ radio over fibre using UF-OFDM with optical heterodyning," in Proc. Int. Top. Meeting Microw. Phot., 2017, Paper Th2.4.

[35] H.-Y. Wang et al., "Tri-color optical transmitter with embedding 28-GHz millimeter-wave carrier for $5 \mathrm{G}$ mobile over fiber," in Proc. Conf. Lasers . Electro-Opt., 2017, Paper SM1O.1.

[36] Y. Tian et al., "Demonstration of non-orthogonal multiple access scheme using multilevel coding without successive interference cancellation with $60 \mathrm{GHz}$ radio-over-fiber fronthaul," in Proc. Opt. Fiber Commun. Conf., 2018, Paper Tu3J.4.

[37] E. Ruggeri et al., "Fiber wireless A-RoF/IFoF uplink of $0.4 \mathrm{~Gb} / \mathrm{s}$ 16-QAM and $0.6 \mathrm{~Gb} / \mathrm{s}$ QPSK over a 32-Element $60 \mathrm{GHz}$ phased array antenna for 5 G fronthaul networks," in Proc. Int. Top. Meeting Microw. Phot., 2019, Paper Th1.4.

[38] X. Li et al., "Real-time demonstration of over 20 Gbps V- and W-Band wireless transmission capacity in one OFDM-RoF system," in Proc. Opt. Fiber Commun. Conf., 2017, Paper M3E.3. 